McGranahan, Devan Allen, Torre J. Hovick, R. Dwayne Elmore, David M. Engle, Samuel D. Fuhlendorf, Stephen L.

Winter, James R. Miller, and Diane M. Debinski. 2016. Temporal variability in aboveground biomass decreases as spatial heterogeneity increases. Ecology 97(3):555-560.http://dx.doi.org/10.1890/15-0906

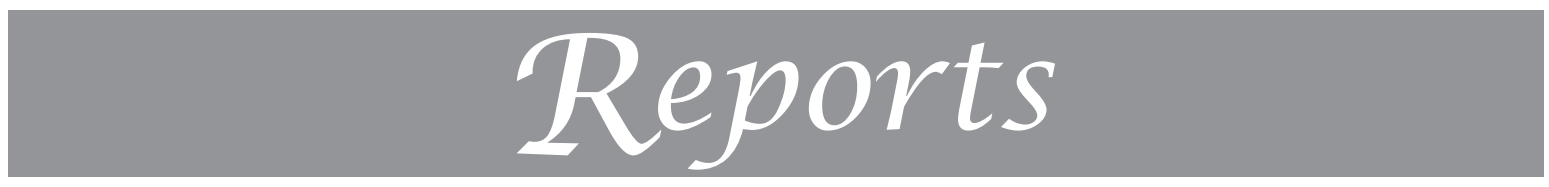

\title{
Temporal variability in aboveground plant biomass decreases as spatial variability increases
}

\author{
Devan Allen McGranahan,,${ }^{1,5}$ Torre J. Hovick, ${ }^{1}$ R. Dwayne Elmore, ${ }^{2}$ David M. Engle, ${ }^{2}$ \\ Samuel D. Fuhlendorf, ${ }^{2}$ Stephen L. Winter, ${ }^{2}$ James R. Miller,${ }^{3}$ and Diane M. Debinski ${ }^{4}$ \\ ${ }^{1}$ School of Natural Resource Sciences-Range Science Program, North Dakota State University, Fargo, \\ North Dakota 58108-6050, USA \\ ${ }^{2}$ Department of Natural Resource Ecology and Management, Oklahoma State University, Stillwater, Oklahoma 74078, USA \\ ${ }^{3}$ Department of Natural Resources and Environmental Sciences, University of Illinois, Urbana, Illinois 61801, USA \\ ${ }^{4}$ Department of Ecology, Evolution and Organismal Biology, Iowa State University, Ames, Iowa 50010, USA
}

\begin{abstract}
Ecological theory predicts that diversity decreases variability in ecosystem function. We predict that, at the landscape scale, spatial variability created by a mosaic of contrasting patches that differ in time since disturbance will decrease temporal variability in aboveground plant biomass. Using data from a multi-year study of seven grazed tallgrass prairie landscapes, each experimentally managed for one to eight patches, we show that increased spatial variability driven by spatially patchy fire and herbivory reduces temporal variability in aboveground plant biomass. This pattern is associated with statistical evidence for the portfolio effect and a positive relationship between temporal variability and functional group synchrony as predicted by metacommunity variability theory. As disturbance from fire and grazing interact to create a shifting mosaic of spatially heterogeneous patches within a landscape, temporal variability in aboveground plant biomass can be dampened. These results suggest that spatially heterogeneous disturbance regimes contribute to a portfolio of ecosystem functions provided by biodiversity, including wildlife habitat, fuel, and forage. We discuss how spatial patterns of disturbance drive variability within and among patches.
\end{abstract}

Key words: asynchrony; diversity-stability relationship; ecosystem function; fire-grazing interaction; metacommunity variability; patch burn-grazing; portfolio effect; pyric herbivory.

\section{INTRODUCTION}

Biodiversity drives many ecological processes and services (Naeem 2002, Isbell et al. 2011). The diversity-stability hypothesis predicts diverse plant communities demonstrate greater stability in biomass production and has received meta-analytical support (McNaughton 1977, Tilman et al. 2006, Ives and Carpenter 2007, Campbell et al. 2011, Gross et al. 2014). Most ecological investigations of diversity-stability interpret stability as constancy, in which an ecosystem function such as primary production changes little through time or across space (King and Pimm 1983, Caldeira et al. 2005). Defined as such, stability can be measured as variability among observational units (Caldeira et al. 2005, Ives and Carpenter 2007).

Manuscript received 17 May 2015; revised 20 October 2015; accepted 23 November 2015. Corresponding Editor: P. B. Adler.

${ }^{5}$ E-mail:mcgranah@alumni.grinnell.edu
The portfolio effect and asynchrony are two mechanisms behind the diversity-stability hypothesis in plant communities (Isbell et al. 2009). In the portfolio effect, variability among community constituents averages out as the number of constituents in the community increases; i.e., as the community diversifies, constancy increases (Doak et al. 1998). The portfolio effect is attributed to both statistical inevitability and biological differences among species, although the relative contributions of each can vary among systems (Tilman et al. 1998). Biological differences create asynchrony: the less synchronized fluctuations in production are among community constituents, the more likely community-level production is stable (Loreau and de Mazancourt 2008).

While the diversity-stability hypothesis was developed and mostly tested at single spatial scales in small plots, the metacommunity concept scales productionstabilizing processes to the landscape level. A metacommunity is a set of local patches, defined as discrete 
areas of habitat that hold local communities (Leibold et al. 2004). Distinguishing spatially discrete local communities from extensive metacommunities invites the hypothesis that beta diversity-i.e., differences among discrete patches or local communities - increases broadscale stability of species composition (Zimmerman et al. 2010, Aragón et al. 2011, Pasari et al. 2013). Consistent with diversity-stability theory, portfolio effects and asynchrony mechanisms contribute to metacommunity stability (Downing et al. 2014, Thompson et al. 2015). Along with individual species, functional group diversity also enhances ecosystem stability (Bai et al. 2004, Gherardi and Sala 2015) but has not been tested in the metacommunity framework.

Variability itself can be partitioned into alpha, beta, and gamma components to relate dynamics within and across local communities to metacommunity stability. With a focus on plant biomass production, Wang and Loreau (2014) demonstrate how greater variability within local communities (alpha variability) decreases metacommunity stability (gamma variability), while greater variability across local communities (beta variability) increases metacommunity stability. Distinct biotic processes drive each component: species-specific dynamics drive alpha variability, while patch-level factors such as number of patches, unevenness across patches, and community similarity between patches drive beta variability (Wang and Loreau 2014).

We hypothesize that greater spatial unevenness of biomass production across local communities reduces temporal variability in metacommunity biomass production at the landscape scale. Our data come from seven experimental landscapes in the U.S. Great Plains managed with fire and herbivory to create a gradient of patchiness. The interaction of spatially heterogeneous fire and herbivory creates contrast between spatially discrete patches of grassland vegetation (McGranahan et al. 2012). This is similar to the relationship between spatial heterogeneity and beta variability (Wang and Loreau 2014), but neither the relationship between patch contrast and temporal stability in biomass production nor a gradient of patchiness have been experimentally explored. We expect to find (1) temporal variability in aboveground biomass will decrease as spatial variability increases; (2) a slope $>1$ in the linear relationship between the logarithm of variance and the logarithm of mean aboveground biomass (Doak et al. 1998, Tilman et al. 1998); and (3) a positive relationship between temporal variability and functional group synchrony, as evidence for the portfolio effect and functional group asynchrony as stabilizing mechanisms at the metacommunity scale, respectively.

\section{Materials And Methods \\ Study location and design}

Our study was located at The Nature Conservancy's 16 000-ha Tallgrass Prairie Preserve in northeastern
Oklahoma, USA. The Preserve is dominated by Andropogon gerardii Vitman, Schizachyrium scoparium Nash, Panicum virgatum L., and Sorghastrum nutans (L.) Nash. Common forbs include Ambrosia, Asclepias, Helianthus, and Veronia spp.; shrubs are mostly Rubus spp. The temperate climate is characterized by hot summers and cold winters. Growing season (April-October) rainfall averaged $659 \pm 116 \mathrm{~mm}$ (mean \pm SE) for the study period 2011-2013.

The study consisted of seven experimental landscapes (430-980 ha) in which we manipulated the spatial pattern of fire to create different levels of patchiness, or landscape-level heterogeneity (Hovick et al. 2015). Fire pattern was manipulated by burning a different proportion of each landscape each year to create a gradient of heterogeneity, i.e., spatial variability, among spatially discrete patches. Fire-return intervals ranged from 1 to $4 \mathrm{yr}$ and three landscapes with four, six, and eight patches included both spring and summer burns every year. The seven spatial-temporal fire regimes that resulted are as follows: (1) a single patch burned each spring; (2) two patches, each burned every other spring; (3) three patches, each burned every third spring; (4) four patches, each burned every fourth spring; (5) four patches, one burned every other spring and one burned every other summer; (6) six patches, one burned every third spring and one burned every third summer; and (7) eight patches, one burned every fourth spring and one burned every fourth summer. Appendix S1: Fig. S1 reproduces the design schematic presented by Hovick et al. (2015). Within each experimental landscape, cattle (Bos taurus) were stocked continuously during the growing season at moderate stocking rates with no interior fencing. Herbivory enhances patch contrast created by spatially heterogeneous fire because herbivores concentrate on the most recently burned patch and maintain low vegetation structure through the growing season (Allred et al. 2011, McGranahan et al. 2012).

We collected vegetation data in mid-June of each growing season from 12 randomly placed plots $>200 \mathrm{~m}$ apart within each landscape (Hovick et al. 2015), which constitute local communities within the metacommunity variability framework (Wang and Loreau 2014). Data at each plot were averaged across 17 measurements of (1) total aboveground plant biomass and (2) canopy cover of plant functional groups. We used visual obstruction, which combines vegetation height and density (Harrell and Fuhlendorf 2002) into a non-destructive sampling technique - to estimate aboveground plant biomass (Vermeire et al. 2002). Specifically, we used a Nudds board, from which visual obstruction is observed at $1 \mathrm{~m}$ height from a distance of $7.5 \mathrm{~m}$ (Nudds 1977). We visually estimated canopy cover of plant functional groups (graminoids, forbs, and shrubs) in $0.5 \mathrm{~m}^{2}$ quadrats using a cover class index (Daubenmire 1959). 


\section{Data analysis}

Random-effect regression to determine variance. - We used crossed random-effect linear regression to determine the variance in aboveground plant biomass attributable to spatial and temporal factors in the sampling design (McGranahan et al. 2012, Winter et al. 2012). Although Wang and Loreau (2014) measure variability as the coefficient of variation $(\mathrm{CV})$, we avoid $\mathrm{CV}$ with our data because $\mathrm{CV}$ scales with the mean while our tested effect, contrast between patch-level biomass, depends on variable means; thus for CV the pattern is overwhelmed by disproportionately minute differences in the mean of the low-biomass most recently burned patch. Our model is based on linear mixed-effect regression, which includes terms for both fixed and random effects, but includes no fixed effect term and is thus employed to model variance structure alone. Our model included a temporal term to represent variation in aboveground biomass across years (gamma variability) and a spatial term to represent variation in aboveground biomass among local communities (beta variability). We modified the custom function RE.var (McGranahan et al. 2015) to extract variance components from a random-effect regression model fit to a Gaussian distribution with the lmer function in the R package lme4 (Bates et al. 2013, R Development Core Team 2013). See the Supplement for script for RE.var and all statistical analyses.

Temporal variability vs. spatial heterogeneity.-To test the hypothesis that temporal (gamma) variability in aboveground biomass declines as spatial (beta) variability increases, we compared variance in the temporal term against variance in the spatial term of crossed randomeffect regression results from each landscape with linear least-squares regression in R. For all least squares regression models, we calculated $95 \%$ confidence intervals with $\mathrm{R}$ function confint and extracted 95\% confidence intervals for regression lines with $\mathrm{R}$ package ggplot2 (Wickham 2009).

Mechanisms of stabilization.-We also tested two interrelated stabilization mechanisms, the portfolio effect and asynchrony (Isbell et al. 2009). A slope greater than one in a linear relationship between the logarithm of variance and the logarithm of mean aboveground biomass represents statistical evidence for the portfolio effect (Doak et al. 1998, Tilman et al. 1998), which we tested with least squares linear regression. To test functional group asynchrony, we used Loreau and de Mazancourt's (2008) synchrony equation, in which the variance of total biomass is divided by the squared sum of variance in the biomass of each functional group. To test for a stabilizing effect of asynchrony at the metacommunity level (i.e., reduced gamma variability), we fit temporal variability against landscape-level synchrony (i.e., beta variability) for each landscape in a least squares linear regression model (Isbell et al. 2009).

\section{RESULTS}

Greater spatial (beta) variability was associated with lower temporal (gamma) variability in aboveground biomass $\left(F_{1,5}=7.35, P=0.04, R^{2}=0.60\right.$; Fig. 1). The $95 \%$ confidence interval for the spatial (beta) variability term extended from -0.01 to -0.44 .

As evidence that the portfolio effect accounted for decreased temporal (gamma) variability in aboveground biomass, variance in aboveground biomass increased with the mean aboveground biomass $\left(F_{1,5}=10.44\right.$, $P=0.02, R^{2}=0.68,95 \% \mathrm{CI}=0.58-5.12$; Fig. 2A). The equation for the linear regression line, $\log ($ variance $)=2.85 \times \log ($ mean $)-5.69$, provides evidence for the portfolio effect because the slope, 2.85 , is greater than 1. In our test of metacommunity functional group asynchrony, temporal (gamma) variability increased as synchrony increased (i.e., beta variability decreased; $F_{1,5}=7.48, P=0.04, R^{2}=0.60,95 \% \mathrm{CI}=16.8-541.5$; Fig. 2B).

\section{Discussion}

Temporal (gamma) variability in aboveground biomass declined with increasing spatial (beta) variability as predicted by metacommunity variability theory (Wang and Loreau 2014). Reduced variability of an ecological property is a measure of stability in that

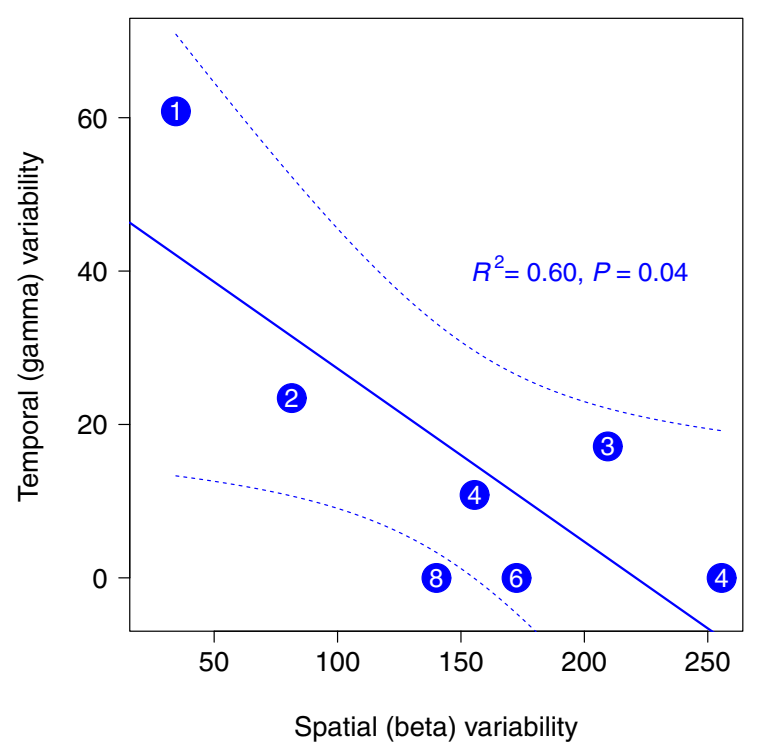

FIG. 1. Temporal (gamma) variability in metacommunity aboveground biomass declines as spatial (beta) variability increases among local communities across seven experimental grassland landscapes in the U.S. Great Plains. Variability measured as variance partitions in landscape-level, crossed random-effect, linear regression models (see Methods and the Supplement). Each symbol denotes a single experimental landscape, and numerals indicate the number of patches into which each landscape was divided. Broken lines denote $95 \%$ confidence intervals for the fitted least-squares linear regression model. 


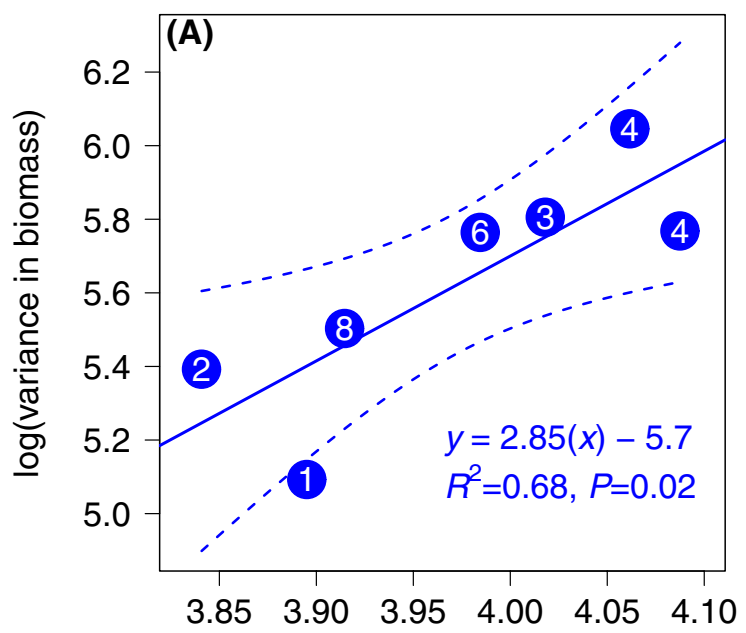

$\log$ (mean biomass)

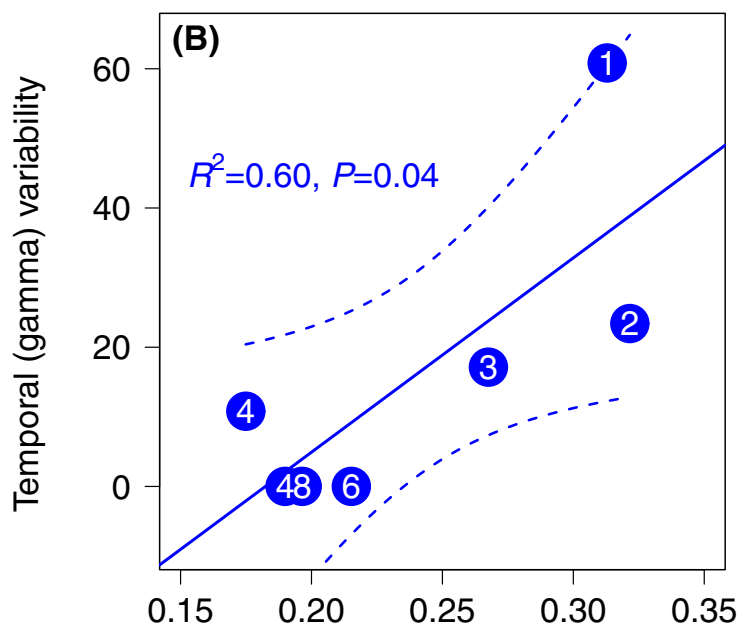

Functional group synchrony

FIG. 2. Two mechanisms by which increased spatial (beta) variability reduced temporal (gamma) variability across seven experimental grassland landscapes in the U.S. Great Plains. (A) Statistical evidence for the portfolio effect as the mechanism behind the metacommunity stability pattern shown in Fig. 1. Biomass in axis labels refers to the sum of aboveground plant biomass from graminoid, forb, and shrub functional groups. Data support the portfolio effect when the slope of the regression equation for $\log ($ variance) vs. $\log ($ mean $)>1$. (B) Temporal variability in aboveground biomass (i.e., metacommunity gamma variability) was greatest in landscapes where metacommunity fluctuations in aboveground biomass by functional group was synchronized (i.e., decreased beta variability); on the converse, temporal (gamma) variability was lowest when fluctuations in aboveground plant biomass were asynchronous among functional groups over time (i.e., increased beta variability). Variability was measured as variance partitions in landscapelevel, crossed random-effect, linear regression models (see Methods and the Supplement). In each panel, symbols denote experimental landscapes, with numerals indicating the number of patches into which each landscape was divided. Broken lines denote $95 \%$ confidence intervals for the fitted least-squares linear regression model. property (Caldeira et al. 2005), and we suggest that spatially heterogeneous patches reinforce a portfolio effect (Doak et al. 1998, Isbell et al. 2009) within a functionally diverse landscape (Fahrig et al. 2011). While compensatory effects among plant functional groups also stabilize productivity (Bai et al. 2004, Gherardi and Sala 2015), the portfolio effect is a main mechanism of stability in mesic grassland (Hallett et al. 2014). Our study is a novel extension of the portfolio effect from plant species to functional groups in a metacommunity context.

Previous studies have examined the diversity-stability hypothesis at plot-level scales several orders of magnitude smaller than our experimental landscapes (e.g., Tilman et al. 2006, Hector et al. 2010). Our study integrates the spatially broad approach of ecosystem ecology into community ecology-based biodiversity research (Loreau 2009) and offers an experimental test of metacommunity variability theory (Wang and Loreau 2014). Specifically, we show greater beta variability reduces gamma variability. Furthermore, our results support a metacommunity portfolio effect driven by statistical probabilities as well as ecological patch dynamics including functional group asynchrony among local communities (Doak et al. 1998, Tilman et al. 1998, Tilman 1999). However, despite the negative, linear trend between temporal and spatial variability, the effect did not constantly increase from landscapes with one to eight patches. Rather, landscapes with four patches tended to occur at the end of each regression gradient opposite landscapes with one or two patches (Figs. 1 and 2), which suggests a diminishing effect of spatial variability beyond four patches. A diminished effect of spatial variability could be due to a trade-off with landscape fragmentation (Fahrig 2003) but further study with manipulated patch size, not just patch number, will be required to decompose these effects.

Our study represents the first time that landscapes driven by pyric herbivory have been explicitly conceptualized as metacommunities. Previously the shifting landscape mosaic created by patches with different times since fire has been described as being driven by the same processes of ecological disturbance and secondary succession that are asynchronous across patches (Fuhlendorf and Engle 2004). Asynchronous patch-level succession explains the diversity observed in spatially heterogeneous landscapes, as a breadth of organisms find specific resources not otherwise available in space or time in spatially homogenous landscapes (reviewed in McGranahan et al. 2013), but offers little to understand how patch-specific dynamics vary across space and through time and thus contribute to landscape-level functionality. Metacommunity theory, however, allows patches to be considered at two spatial scales simultaneously, as spatially discrete communities with independent processes that collectively contribute to landscape-level ecological properties (Loreau 2009). 
Our results support the importance of ecological disturbance as a factor in variability at both fine and broad scales. Although disturbance has been assumed to increase ecosystem variability (Fraterrigo and Rusak 2008), the metacommunity variability framework partitions overall variability into two components: alpha and beta, or within and between local communities, respectively (Wang and Loreau 2014). Under metacommunity variability theory, spatially discrete, ecological disturbance within local communities reduces compositional variability over time at the landscape level (e.g., Fuhlendorf et al. 2006, Zimmerman et al. 2010) by increasing beta variability. We demonstrate this effect for aboveground biomass.

Increasing beta variability by creating spatial heterogeneity, and thus reducing temporal (gamma) variability, in fire-adapted grassland depends on coupled fire and grazing disturbances. Fire shapes the pattern of vegetation, and through quality differences, vegetation shapes the pattern of herbivory. When herbivory increases contrast between patches, beta variability among patches increases; otherwise, low patch contrast can increase alpha variability relative to beta variability and increase overall (gamma) variability in the metacommunity (Adler et al. 2001, Wang and Loreau 2014). It is likely that the same factors that dampen patch contrast despite spatially heterogeneous fire regimes, e.g., invasive species and overgrazing (McGranahan et al. 2012), reduce beta variability and increase the relative effect of within-patch alpha variability. For example, in our study, the single-patch landscape had a spatially homogenous fire pattern and did not induce spatially discrete, patch-level herbivory. The subsequent dispersed pattern of herbivory did not create spatial heterogeneity, consistent with high alpha variability in metacommunity variability theory (Wang and Loreau 2014). This is the dominant pattern of homogenously managed grassland across the US Great Plains (McGranahan et al. 2012), which challenges biodiversity conservation because low patch contrast limits niche opportunities for wildlife and increases temporal species turnover (Hovick et al. 2015).

Biodiversity studies are often motivated by a concern for what biodiversity loss means for ecosystem function, and ours is no different. These results have implications within the context of global environmental change, as increased landscape heterogeneity might buffer ecosystem function. Aboveground biomass relates to a breadth of functions, including wildlife habitat, fuel for fire, and forage for herbivores. Simply stated, heterogeneous landscapes, which include lawnlike patches to deep litter and tall, rank necromass, support greater biological diversity than homogeneous landscapes (McGranahan et al. 2013). And in periods of drought and/or high animal numbers, previously ungrazed, accumulated biomass functions as emergency forage for herbivores (Fynn 2012). Although moribund material is often the lowest-quality forage in the landscape (Sensenig et al. 2010), these patches buffer against starvation, and actually stabilize herbivore populations by balancing forage quality against quantity (Owen-Smith 2004). Many landscapes are nonequilibrium systems in which variation in biomass availability can destabilize ecosystem structure and function, especially if secondary productivity does not respond in kind (Fynn 2012). Our results that increasing spatial heterogeneity buffers against temporal variability suggest heterogeneity can be managed to buffer ecosystem response to global environmental change (Turner 2010).

\section{ACKNOWLEDGMENTS}

We acknowledge the support of USDA-AFRI (\#201085101-20457) and the Oklahoma and North Dakota Agricultural Experiment Stations, and appreciate the contributions of C. Goad, P. Dixon, K. Pazdernick, F. Isbell, T. Parr, and two anonymous reviewers.

\section{Literature Cited}

Adler, P. B., D. A. Raff, and W. K. Lauenroth. 2001. The effect of grazing on the spatial heterogeneity of vegetation. Oecologia 128:465-479.

Allred, B. W., S. D. Fuhlendorf, D. M. Engle, and R. D. Elmore. 2011. Ungulate preference for burned patches reveals strength of fire-grazing interaction. Ecology and Evolution 1:132-144.

Aragón, R., M. Oesterheld, G. Irisarri, and M. Texeira. 2011. Stability of ecosystem functioning and diversity of grasslands at the landscape scale. Landscape Ecology 26:1011-1022.

Bai, Y., X. Han, J. Wu, Z. Chen, and L. Li. 2004. Ecosystem stability and compensatory effects in the Inner Mongolia grassland. Nature 431:181-184.

Bates, D., M. Maechler, B. Bolker, and S. Walker. 2013. lme4: linear mixed-effects models using Eigen and S4. http://CRAN.R-project.org/package $=\operatorname{lme} 4$

Caldeira, M. C., A. Hector, M. Loreau, and J. S. Pereira. 2005. Species richness, temporal variability and resistance of biomass production in a Mediterranean grassland. Oikos 110:115-123.

Campbell, V., G. Murphy, and T. N. Romanuk. 2011. Experimental design and the outcome and interpretation of diversity-stability relations. Oikos 120:399-408.

Daubenmire, R. 1959. A canopy-coverage method of vegetational analysis. Northwest Science 33:43-64.

Doak, D. F., D. Bigger, E. K. Harding, M. A. Marvier, R. E. O'Malley, and D. Thomson. 1998. The statistical inevitability of stability-diversity relationships in community ecology. American Naturalist 151:264-276.

Downing, A. L., B. L. Brown, and M. A. Leibold. 2014. Multiple diversity-stability mechanisms enhance population and community stability in aquatic food webs. Ecology 95:173-184.

Fahrig, L. 2003. Effects of habitat fragmentation on biodiversity. Annual Review of Ecology, Evolution, and Systematics 34:487-515.

Fahrig, L., J. Baudry, L. Brotons, F. G. Burel, T. O. Crist, R. J. Fuller, C. Sirami, G. M. Siriwardena, and J.-L. Martin. 2011. Functional landscape heterogeneity and animal biodiversity in agricultural landscapes. Ecology Letters 14:101-112.

Fraterrigo, J. M., and J. A. Rusak. 2008. Disturbance-driven changes in the variability of ecological patterns and processes. Ecology Letters 11:756-770. 
Fuhlendorf, S. D., and D. M. Engle. 2004. Application of the fire-grazing interaction to restore a shifting mosaic on tallgrass prairie. Journal of Applied Ecology 41:604-614.

Fuhlendorf, S. D., W. C. Harrell, D. M. Engle, R. G. Hamilton, C. A. Davis, and D. M. Leslie Jr. 2006. Should heterogeneity be the basis for conservation? Grassland bird response to fire and grazing. Ecological Applications 16:1706-1716.

Fynn, R. W. S. 2012. Functional resource heterogeneity increases livestock and rangeland productivity. Rangeland Ecology \& Management 65:319-329.

Gherardi, L. A., and O. E. Sala. 2015. Enhanced interannual precipitation variability increases plant functional diversity that in turn ameliorates negative impact on productivity. Ecology Letters 18:1293-1300.

Gross, K., B. J. Cardinale, J. W. Fox, A. Gonzalez, M. Loreau, H. Wayne Polley, P. B. Reich, and J. van Ruijven. 2014. Species richness and the temporal stability of biomass production: a new analysis of recent biodiversity experiments. American Naturalist 183:1-12.

Hallett, L. M., et al. 2014. Biotic mechanisms of community stability shift along a precipitation gradient. Ecology 95:1693-1700.

Harrell, W. C., and S. D. Fuhlendorf. 2002. Evaluation of habitat structural measures in a shrubland community. Journal of Range Management 55:488-493.

Hector, A., Y. Hautier, P. Saner, L. Wacker, R. Bagchi, J. Joshi, M. Scherer-Lorenzen, E. M. Spehn, E. Bazeley-White, and M. Weilenmann. 2010. General stabilizing effects of plant diversity on grassland productivity through population asynchrony and overyielding. Ecology 91:2213-2220.

Hovick, T. J., R. D. Elmore, S. D. Fuhlendorf, D. M. Engle, and R. G. Hamilton. 2015. Spatial heterogeneity increases diversity and stability in grassland bird communities. Ecological Applications 25:662-672.

Isbell, F. I., H. W. Polley, and B. J. Wilsey. 2009. Biodiversity, productivity and the temporal stability of productivity: patterns and processes. Ecology Letters 12:443-451.

Isbell, F., et al. 2011. High plant diversity is needed to maintain ecosystem services. Nature 477:199-202.

Ives, A. R., and S. R. Carpenter. 2007. Stability and diversity of ecosystems. Science 317:58-62.

King, A. W., and S. L. Pimm. 1983. Complexity, diversity, and stability: a reconciliation of theoretical and empirical results. American Naturalist 122:229-239.

Leibold, M. A., et al. 2004. The metacommunity concept: a framework for multi-scale community ecology. Ecology Letters 7:601-613.

Loreau, M. 2009. Linking biodiversity and ecosystems: towards a unifying ecological theory. Philosophical Transactions of the Royal Society B 365:49-60.

Loreau, M., and C. de Mazancourt. 2008. Species synchrony and its drivers: neutral and nonneutral community dynamics in fluctuating environments. American Naturalist 172:E48-E66.

McGranahan, D. A., D. M. Engle, S. D. Fuhlendorf, S. J. Winter, J. R. Miller, and D. M. Debinski. 2012. Spatial heterogeneity across five rangelands managed with pyric-herbivory. Journal of Applied Ecology 49:903-910.
McGranahan, D. A., D. M. Engle, S. D. Fuhlendorf, S. L. Winter, J. R. Miller, and D. M. Debinski. 2013. Inconsistent outcomes of heterogeneity-based management underscore importance of matching evaluation to conservation objectives. Environmental Science \& Policy 31:53-60.

McGranahan, D. A., R. Burgdorf, and K. P. Kirkman. 2015. Epichloae infection in a native South African grass, Festuca costata Nees. Plant Biology 17:914-921.

McNaughton, S. J. 1977. Diversity and stability of ecological communities: a comment on the role of empiricism in ecology. American Naturalist 111:515-525.

Naeem, S. 2002. Ecosystem consequences of biodiversity loss: the evolution of a paradigm. Ecology 83:1537-1552.

Nudds, T. D. 1977. Quantifying the vegetative structure of wildlife cover. Wildlife Society Bulletin 5:113-117.

Owen-Smith, N. 2004. Functional heterogeneity in resources within landscapes and herbivore population dynamics. Landscape Ecology 19:761-771.

Pasari, J. R., T. Levi, E. S. Zavaleta, and D. Tilman. 2013. Several scales of biodiversity affect ecosystem multifunctionality. Proceedings of the National Academy of Sciences USA 110:15163.

R Development Core Team. 2013. R: a language and environment for statistical computing. R Foundation for Statistical Computing, Vienna, Austria. http://www.R-project.org/

Sensenig, R. L., M. W. Demment, and E. A. Laca. 2010. Allometric scaling predicts preferences for burned patches in a guild of East African grazers. Ecology 91:2898-2907.

Thompson, P. L., B. E. Beisner, and A. Gonzalez. 2015. Warming induces synchrony and destabilizes experimental pond zooplankton metacommunities. Oikos 124:1171-1180.

Tilman, D. 1999. The ecological consequences of changes in biodiversity: a search for general principles. Ecology 80:1455-1474.

Tilman, D., C. L. Lehman, and C. E. Bristow. 1998. Diversity-stability relationships: statistical inevitability or ecological consequence? American Naturalist 151:277-282.

Tilman, D., P. B. Reich, and J. M. Knops. 2006. Biodiversity and ecosystem stability in a decade-long grassland experiment. Nature 441:629-632.

Turner, M. G. 2010. Disturbance and landscape dynamics in a changing world. Ecology 91:2833-2849.

Vermeire, L. T., A. C. Ganguli, and R. L. Gillen. 2002. A robust model for estimating standing crop across vegetation types. Journal of Range Management 55:494-497.

Wang, S., and M. Loreau. 2014. Ecosystem stability in space: $\alpha, \beta$ and $\gamma$ variability. Ecology Letters 17:891-901.

Wickham, H. 2009. ggplot2: elegant graphics for data analysis. Springer, New York, New York, USA.

Winter, S. L., S. D. Fuhlendorf, C. L. Goad, C. A. Davis, K. R. Hickman, and D. M. Leslie Jr. 2012. Restoration of the fire-grazing interaction in Artemisia filifolia shrubland. Journal of Applied Ecology 49:242-250.

Zimmerman, J. K., L. S. Comita, J. Thompson, M. Uriarte, and N. Brokaw. 2010. Patch dynamics and community metastability of a subtropical forest: compound effects of natural disturbance and human land use. Landscape Ecology 25:1099-1111.

\section{SUPPORTING INFORMATION}

Additional supporting information may be found in the online version of this article at http://onlinelibrary.wiley.com/ doi/10.1890/15-0906.1/suppinfo 